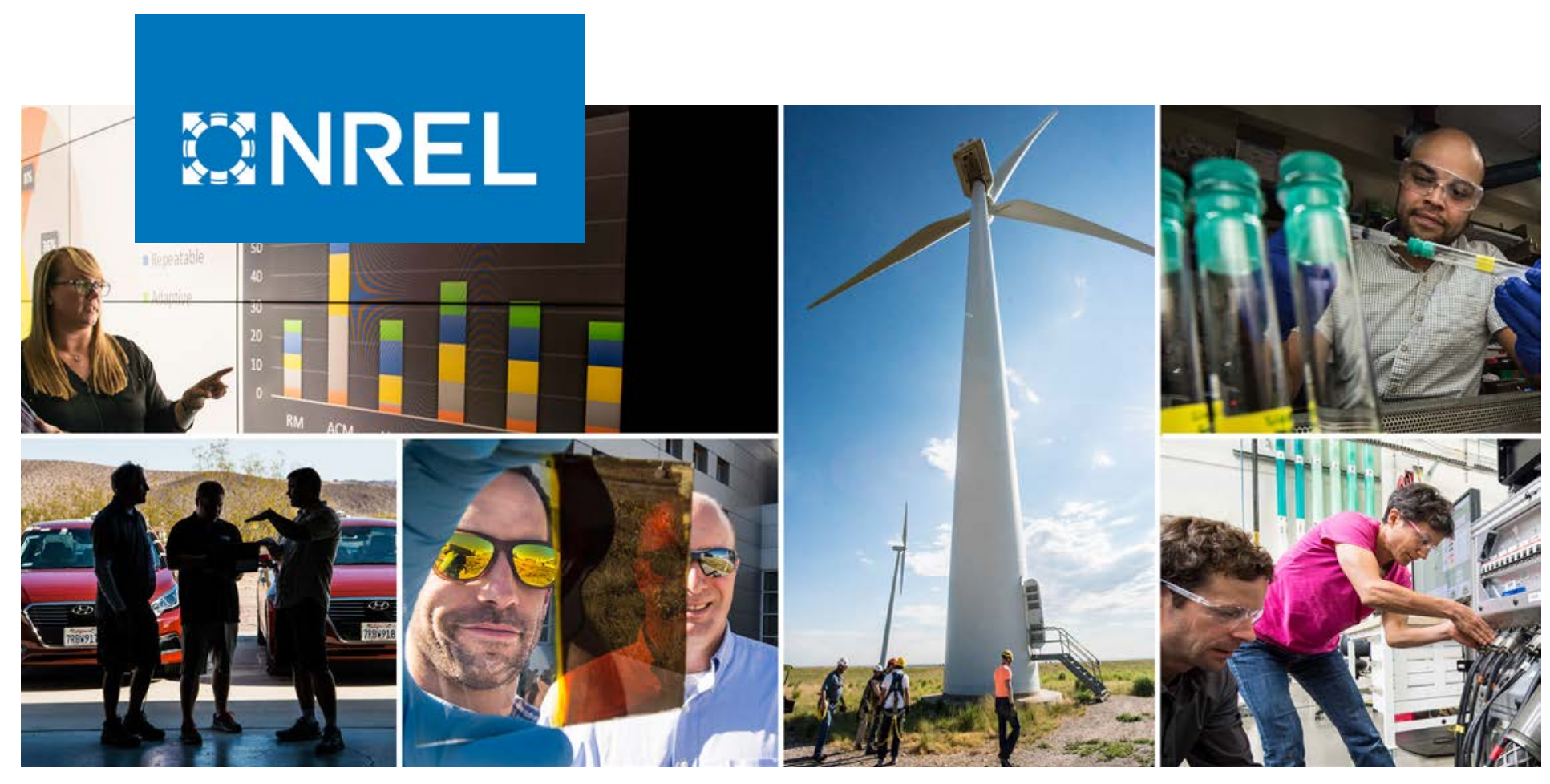

\title{
Validation of Wind Power Plant Modeling Approaches in Complex Terrain
}

\section{Preprint}

Eliot Quon, Paula Doubrawa, Jennifer Annoni, Nicholas Hamilton, and Matthew Churchfield

National Renewable Energy Laboratory

Presented at the American Institute of Aeronautics and Astronautics

SciTech Forum

San Diego, California

January 7-11, 2019

NREL is a national laboratory of the U.S. Department of Energy

Office of Energy Efficiency \& Renewable Energy

Operated by the Alliance for Sustainable Energy, LLC

This report is available at no cost from the National Renewable Energy Laboratory (NREL) at www.nrel.gov/publications.

\section{Conference Paper}

NREL/CP-5000-72706

May 2019 


\title{
GNREL
}

\section{Validation of Wind Power Plant Modeling Approaches in Complex Terrain}

\section{Preprint}

\author{
Eliot Quon, Paula Doubrawa, Jennifer Annoni,
} Nicholas Hamilton, and Matthew Churchfield

National Renewable Energy Laboratory

\section{Suggested Citation}

Quon, Eliot, Paula Doubrawa, Jennifer Annoni, Nicholas Hamilton, and Matthew Churchfield. 2019. Validation of Wind Power Plant Modeling Approaches in Complex Terrain: Preprint. Golden, CO: National Renewable Energy Laboratory. NREL/CP-5000-72706.

https://www.nrel.gov/docs/fy19osti/72706.pdf.

NREL is a national laboratory of the U.S. Department of Energy Office of Energy Efficiency \& Renewable Energy Operated by the Alliance for Sustainable Energy, LLC

This report is available at no cost from the National Renewable Energy Laboratory (NREL) at www.nrel.gov/publications.

Contract No. DE-AC36-08GO28308
Conference Paper NREL/CP-5000-72706 May 2019

National Renewable Energy Laboratory 15013 Denver West Parkway Golden, CO 80401 303-275-3000 • www.nrel.gov 


\section{NOTICE}

This work was authored by the National Renewable Energy Laboratory, operated by Alliance for Sustainable Energy, LLC, for the U.S. Department of Energy (DOE) under Contract No. DE-AC36-08GO28308. Funding provided by the U.S. Department of Energy Office of Energy Efficiency and Renewable Energy Wind Energy Technologies Office. The views expressed herein do not necessarily represent the views of the DOE or the U.S. Government. The U.S. Government retains and the publisher, by accepting the article for publication, acknowledges that the U.S. Government retains a nonexclusive, paid-up, irrevocable, worldwide license to publish or reproduce the published form of this work, or allow others to do so, for U.S. Government purposes.

This report is available at no cost from the National Renewable Energy Laboratory (NREL) at www.nrel.gov/publications.

U.S. Department of Energy (DOE) reports produced after 1991 and a growing number of pre-1991 documents are available free via www.OSTI.gov.

Cover Photos by Dennis Schroeder: (clockwise, left to right) NREL 51934, NREL 45897, NREL 42160, NREL 45891, NREL 48097, NREL 46526.

NREL prints on paper that contains recycled content. 


\title{
Validation of Wind Power Plant Modeling Approaches in Complex Terrain
}

\author{
Eliot Quon, Paula Doubrawa, Jennifer Annoni, Nicholas Hamilton, and Matthew Churchfield \\ National Renewable Energy Laboratory, Golden, Colorado, 80401, USA
}

\begin{abstract}
The effect of terrain on wind-power-plant aerodynamics is often neglected due to the complexity of terrain-atmosphere-turbine interactions and the computational effort required to adequately resolve the relevant physics. In this work, the effects of topography on plant performance are investigated using a comprehensive set of measurements from the Wind Forecast Improvement Project 2 and three modeling approaches operating at different levels of fidelity. A high-fidelity approach consists of large-eddy simulations on a terrain-resolving mesh to produce unsteady, heterogeneous flow that is sensitive to the local topography, with turbines modeled as actuator lines. In comparison, an engineering approach makes use of a steady-state wake model that has been modified to account for terrain effects. An intermediate level of fidelity is achieved through the use of a multiphysics model that couples wake dynamics to the aeroelastic response of each wind turbine and uses inflow from a large-eddy simulation. This paper details a case study of nine turbines in a near-neutral atmospheric boundary layer after a wind-speed ramp. In situ and remote-sensing measurements upstream of the wind plant are used to prescribe initial and boundary conditions to the models. Wind turbine power measurements are used to assess the ability of the different models to capture the effects of terrain on turbine wake dynamics and therefore power production in an array. The study demonstrates that accounting for unsteady turbulent inflow and averaging over time periods longer than 10 minutes are necessary for realistic predictions of mean power output, due to unsteady, local variations in wind speed and direction. Engineering models that account for local uphill terrain steepness may not be sufficient in all cases as favorable pressure-gradient effects may be overestimated and downwind terrain-induced vertical wake displacement is neglected.
\end{abstract}

\section{Introduction}

Wind power plants have proven to be a reliable, cost-effective source of renewable energy, and are key components of the United States' renewable energy portfolio. The U.S. Department of Energy (DOE) has reported that $20 \%$ of the U.S. end-use demand could be satisfied by wind energy alone by 2030 , and $35 \%$ by 2050 [1]. To this end, modern wind plants must operate more intelligently and efficiently than before. The performance and efficiency of a wind plant are largely dictated by layout, wind turbine selection, controls, and atmospheric conditions. Plant layout is of importance because a power-producing wind turbine will have a turbulent, low-speed wake that persists for some distance downstream. Therefore, the arrangement of turbines within the plant determines the degree to which turbines can interact aerodynamically. The negative impact of an upstream turbine wake on downstream turbine performance may be mitigated using controls. Wake steering, for instance, uses yaw misalignment to redirect turbine wakes and minimize downstream rotor waking [2]. The dynamics of these turbine wakes - including the extent to which they meander and the distance at which they recover free-stream flow statistics - as well as the degree to which the wake trajectories may be controlled, are modulated by atmospheric conditions. Predicting the performance of wind turbines is further complicated by nonflat terrain, in which speedup on the windward side of hills and terrain-added turbulence may not be reliably predicted. Neglecting terrain effects can underestimate power reduction for downstream turbines and ultimately overestimate wind plant performance [3]. Furthermore, terrain-induced mean or time-dependent lateral or vertical deflection of the wakes adds another layer of complexity to the consideration of wake management and control. These challenges have motivated recent experimental and computational investigations [4]. 
Characterizing atmospheric conditions from measurements for simulation is nontrivial 5$]$ and accurate mesoscale modeling of the large-scale background conditions driving the flow through a wind plant remains a challenge. To address the challenges of numerical weather prediction, DOE and the National Oceanographic and Atmospheric Administration have sponsored the ongoing Weather Forecast Improvement Project (WFIP) project 6. The second phase of WFIP (WFIP2), which lasted 18 months ending in March 2017, focused on understanding the atmospheric processes that affect wind forecasts in complex terrain. WFIP2 yielded a plethora of coordinated observations from meteorological towers (met masts), surface meteorological stations (met stations), and remote-sensing devices. Data were collected in the Columbia River Gorge in the northwestern United States and include supervisory control and data acquisition (SCADA) output from a number of wind plants in the region. These data offer a unique opportunity to evaluate models of varying fidelity for validation purposes under complex inflow conditions, with measurements of the local inflow and power output from multiple wind plants. Of particular interest are wind farms near to the WFIP2 Physics Site. The site was instrumented with met masts and met stations at 12 locations within a $7.1 \mathrm{~km}^{2}$ area (Fig. 1).

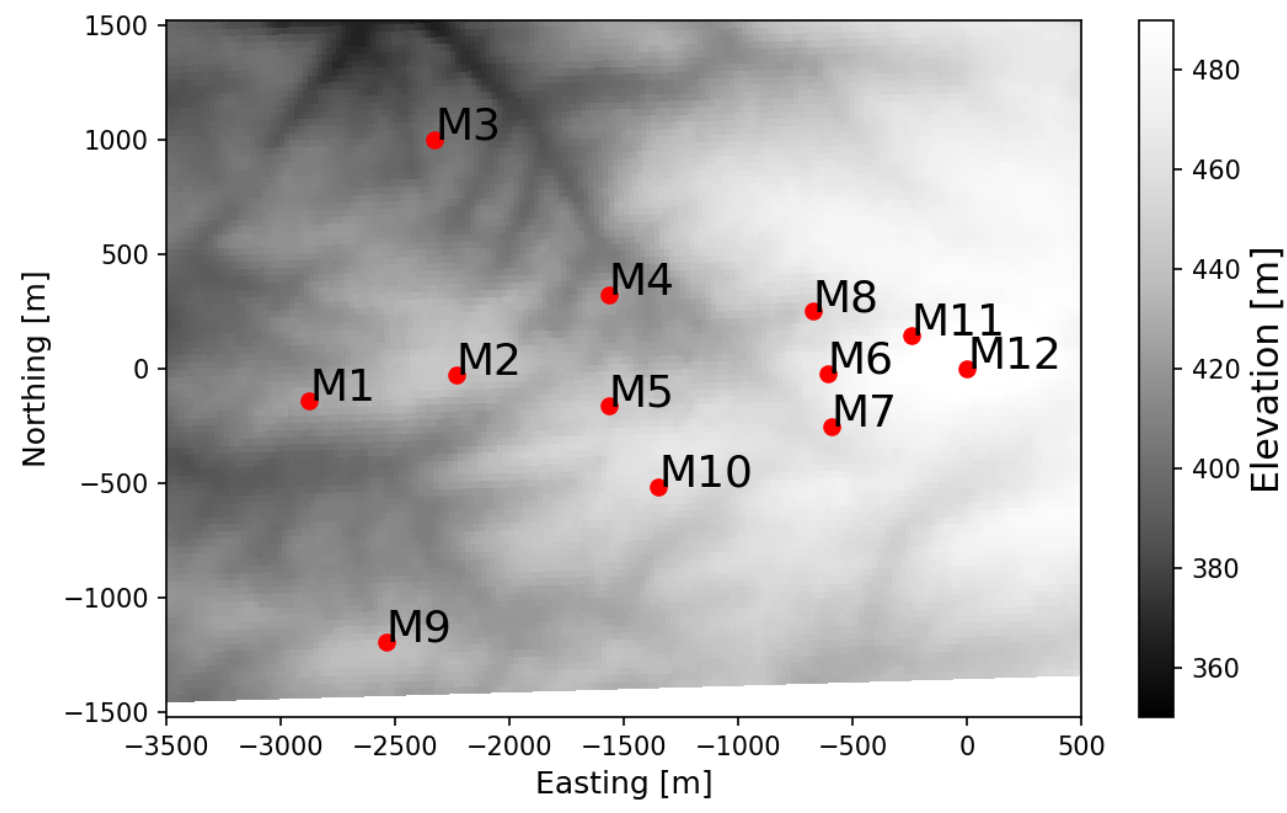

Figure 1. Modeled topography and locations of WFIP2 met masts.

Table 1. WFIP2 Physics Site instrumentation.

\begin{tabular}{clll}
\hline Location & Instrument Class & Measurements & Measurement Height(s) \\
\hline M1 & sodar & wind profile & $40-200 \mathrm{~m}$ \\
M1 & met mast & wind, temperature, and fluxes & 3 and $10 \mathrm{~m}$ \\
M2 & met mast & wind, temperature, and fluxes & 3,10 , and $17 \mathrm{~m}$ \\
M6 & met mast & wind, temperature, and fluxes & 3,10 , and $21 \mathrm{~m}$ \\
M7 & met station & wind, temperature, and solar radiation & $3 \mathrm{~m}$ \\
M11 & met mast & wind, temperature, and fluxes & 3 and $10 \mathrm{~m}$ \\
M12 & met mast & wind and temperature & 50 and $80 \mathrm{~m}$ \\
\hline
\end{tabular}

Typical engineering tools used to calculate wind plant performance assume steady inflow or turbulent inflow with homogeneous, stationary turbulence. These tools empirically model the effects of turbulence and terrain, for example, in the form of a wake expansion parameter 7 . More realistic inflow to the wind plant has a heterogeneous, nonstationary turbulence field driven by mesoscale atmospheric dynamics. Surfaces affect the inflow through topography, variable roughness, or radiative forcing. In addition, weather phenomena, such as frontal passages, cold pools, and large-scale advection, can affect the wind-plant operating environment. Given these complex, evolving conditions, even high-fidelity large-eddy simulations (LES) of 
the atmospheric boundary layer (ABL) may neglect key physics for a given set of simulation conditions. For example, generating LES-resolved turbulence with a canonical precursor (using a strong recycling approach on a separate periodic simulation domain [8]) carries the assumption that the ABL is in a stationary, quasi-equilibrium state. To simulate more realistic conditions, nonstationarity may be forced through timevarying boundary conditions and/or internal forcing terms in the governing equations [9 10]. Most of the aforementioned sources of heterogeneity may be introduced using the boundary-condition or internal-forcing approach except for complex terrain effects.

Much of the previous high-fidelity research and analysis of wind plants in complex terrain was performed with Reynolds-averaged Navier-Stokes (RANS) solvers rather than LES [11]. However, high-fidelity LES is needed to capture turbulent motions in the ABL and accurately simulate the wind plant operating environment. A RANS study considering an idealized Gaussian hill has shown that terrain modifies the wake geometry in a nonlinear way; in other words, the wake from a turbine situated atop a hill is not the same as the superposition of flow over the hill without the turbine with the wake of a turbine on flat terrain [11]. A more recent study demonstrated that, compared to a wind-tunnel experiment, LES is able to accurately capture both the flow speedup over a Gaussian hill as well as the wake velocity deficit and added turbulence due to a turbine on top of that hill [12]. Another recent LES study highlights the computational challenges of modeling realistic complex terrain: when using a segregated pseudo-spectral flow solver, the cost to solve the pressure Poisson equation increases substantially with terrain complexity [4]. Other complex-terrain research has focused on the intricacies of LES numerical methods, including subgrid-scale modeling and the terrain representation. These modeling decisions can affect the size, shape, and location of recirculation zones on the lee side of a hill [13].

In the present study, we apply three models of increasing fidelity to evaluate the effects of complex terrain on turbines in the Columbia River Gorge, focusing on a 1-hour period following a wind speed ramp. During this 1-hour period, atmospheric conditions (described in Section II) were approximately constant. LES predictions of the heterogeneous flow field were validated against field measurements at the WFIP2 Physics Site and individual turbine power predictions from the three different models (detailed in Section III) were validated against the average power produced during the period of interest.

\section{Site Characterization}

The region of interest near the Columbia River Gorge and the subset of wind turbines under investigation are illustrated in Fig. 2 Locations of both the wind turbines and instrument sites are superimposed on the terrain, which were extracted from Shuttle Radar Topography Mission satellite data [14]. Table 1 summarizes the measurements from the WFIP2 Physics Site that have been used in this study.

\section{II.A. Terrain}

The current study builds on previous wind farm LES studies in flat terrain (e.g., Ref. [5 15]). Modeling challenges in this work not only include resolving the uphill inflow and downhill outflow from the turbines, but also the flow over and around additional upwind flow features. Figures 1 and 2 identify the locations of WFIP2 measurements and turbines, respectively, and illustrate the terrain in the region. Within this study area, $25 \%$ of the terrain has a slope between $5^{\circ}$ and $15^{\circ}$. Figure $3(\mathrm{a})$ illustrates the terrain upwind and downwind of each of the wind turbines (WTs) in the front row (WT 1-5), whereas Fig 3(b) illustrates the terrain for the back row (WT 6-9). WT 1, for instance, is situated $0.7 D$ above a gulch located $6 D$ upwind, assuming westerly flow. It is important to note that reference measurements from the Physics Site were not taken from flat terrain (Fig. 3(c)]. Terrain complexity, therefore, also poses challenges for the inflow validation effort. For a realistic LES turbulence field, observations from WFIP2 may correspond to simulated wind profiles that are offset from the actual measurement location due to spatial variability.

\section{II.B. Atmospheric Conditions}

Figure 4 shows observations from WFIP2 on November 21, 2016, used to characterize the wind plant inflow. This particular date is a case study from the DOE Mesoscale-to-Microscale Coupling project [16], selected based on meteorological interests that included a topographical wake from Mount Hood and mountain waves, both observed in mesoscale simulation. The reported met-mast measurements are from the 80-m tower at M12 (Fig. 1), which has four sonic anemometers: two at $50 \mathrm{~m}$ and two at $80 \mathrm{~m}$ above ground. In addition, 


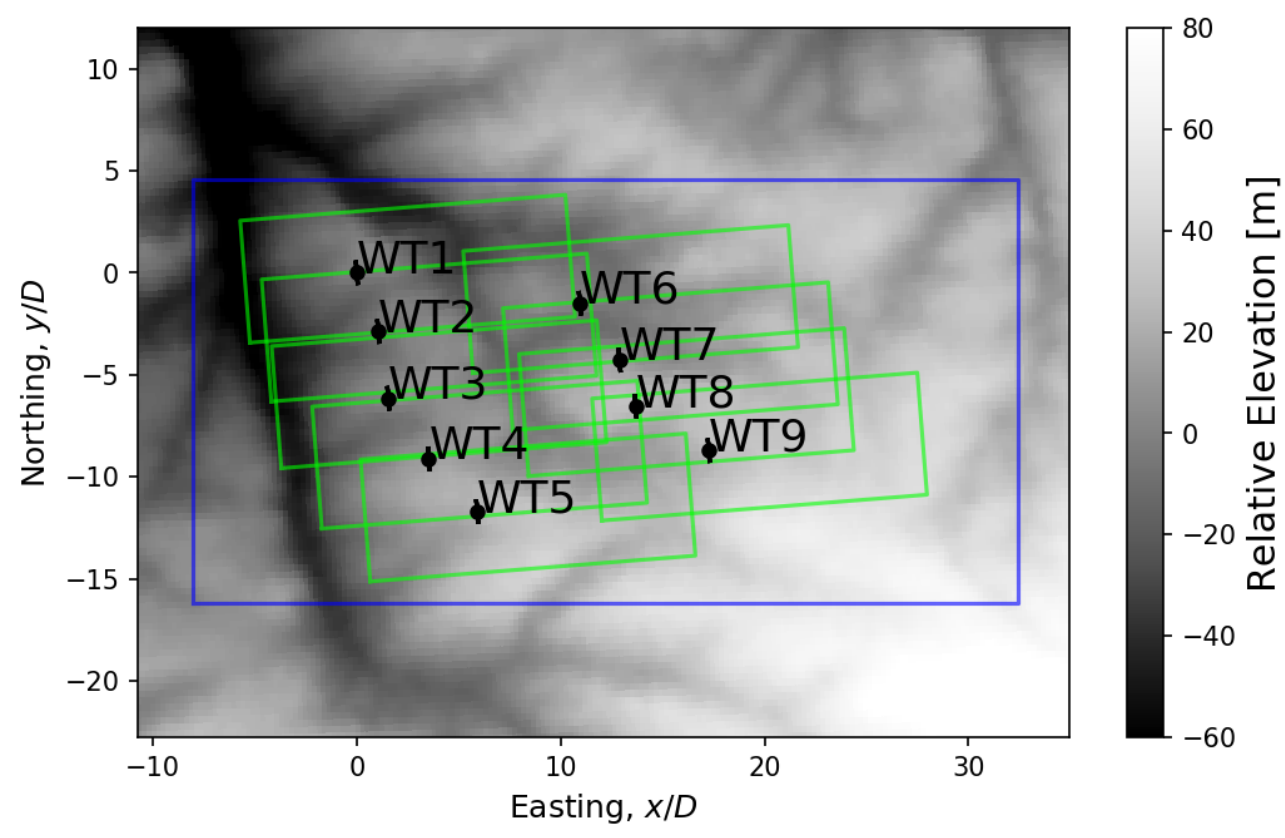

Figure 2. Modeled topography and locations of simulated wind turbines (WTs) relative to turbine 1; the FAST.Farm domain and high-resolution LES regions are outlined in blue and green, respectively.

met stations at M1, M2, M6, and M11 provide detailed heat flux information that can be used to estimate atmospheric stability. Lastly, measurements from a propeller anemometer on a 3-m tower at M7 have been included. The M7 location is also of interest because it has a pyranometer to measure solar radiation, which drives diurnal variations in surface heat transfer and, consequently, atmospheric stability.

Over the course of a day, changes in temperature, wind speed, and power production are coupled. The diurnal cycle is observed in all quantities plotted in Fig. 4 except for wind direction, with the measured wind speed and temperature plateauing within the time period of interest between 2 p.m. and 3 p.m. local time (i.e., 2200-2300 UTC). The downwelling shortwave solar radiation peaks before noon, preceding the peak observed temperatures. Temperatures sharply increase - most notably near to the ground - between 10 and 11 a.m. local time, and the peak temperature occurs after noon at about 1 p.m. With the decrease in solar radiation after noon, temperatures decrease for the remainder of the day starting at 3 p.m.; an hour later the wind speed also decreases.

Our analysis focuses on a group of nine turbines located in complex terrain. The power production (Fig. 5) closely tracks the observed hub-height winds, which predictably lags the diurnal radiative forcing. Clear up-ramp and down-ramp events can be seen at 1 and 4 p.m. local time, respectively. Note that within the period of interest, the back row of selected turbines (WTs 6-9) does not necessarily produce less power, unlike the previous hour between 1 and 2 p.m. local time. The available SCADA data from these turbines also include wind speed and direction from sensors on each wind turbine (plotted in Fig. 4 as circles). Differences between wind speeds reported by the SCADA system and met masts may be attributed to the upwind terrain: the 80-m mast is situated on a hill, whereas the selected turbines are situated in more complex terrain and more than $100 \mathrm{~m}$ lower in elevation.

To further characterize the ABL, we have calculated a number of additional metrics: shear exponent, turbulence intensity (TI), turbulent kinetic energy (TKE), and the Obukhov stability parameter (Fig. 6). Shear and TI are standard descriptors of turbulent inflow to a wind turbine. More importantly, they are often used in wind engineering as proxies for atmospheric stability, which can introduce variations in average turbine power output up to $15 \%$ [17. In general, nighttime stable boundary layers are characterized by high shear, low TI, and low TKE; conversely, daytime convective (unstable) boundary layers have low shear, high TI, and high TKE. Moreover, convective boundary layers tend to extend farther into the atmosphere and have larger turbulence length scales than their stable counterparts, which affects the loads experienced by the turbines operating within the ABL. According to the Obukhov stability parameter, defined as $z / L$, the ABL transitions from unstable to near-neutral during the time period of interest in this study, then to stable 


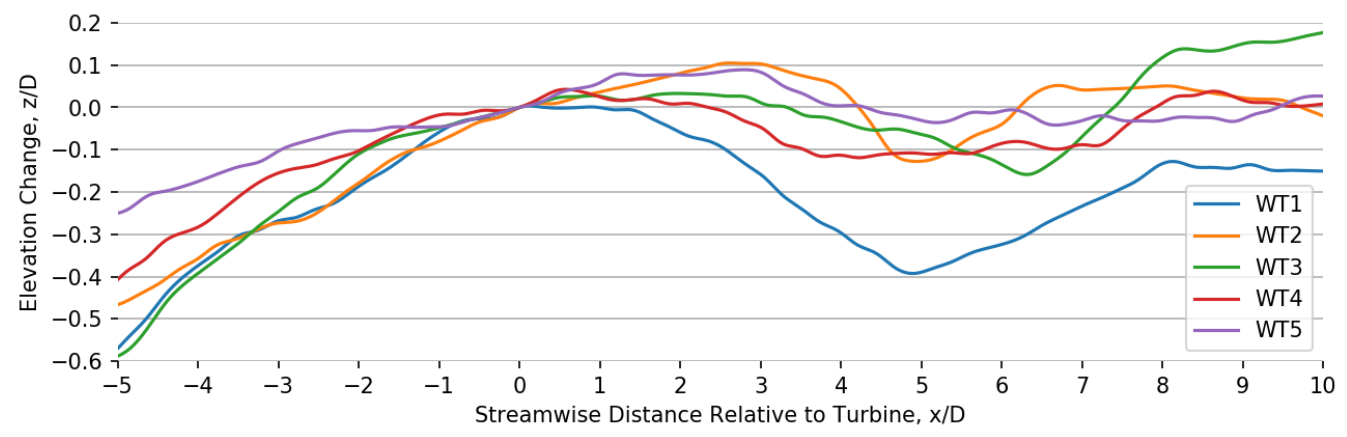

(a) Terrain relative to turbines in the front row, WT 1-5

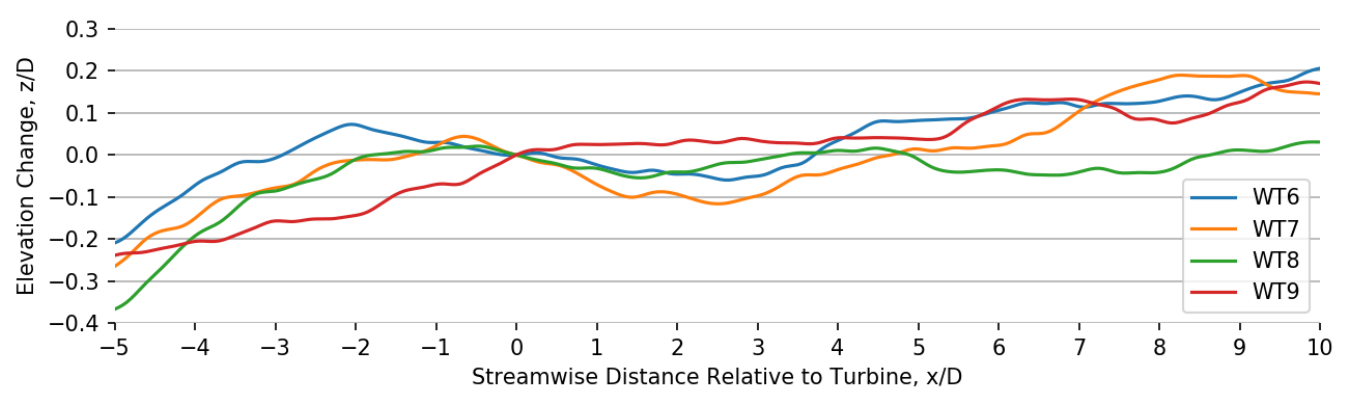

(b) Terrain relative to turbines in the back row, WT 6-9

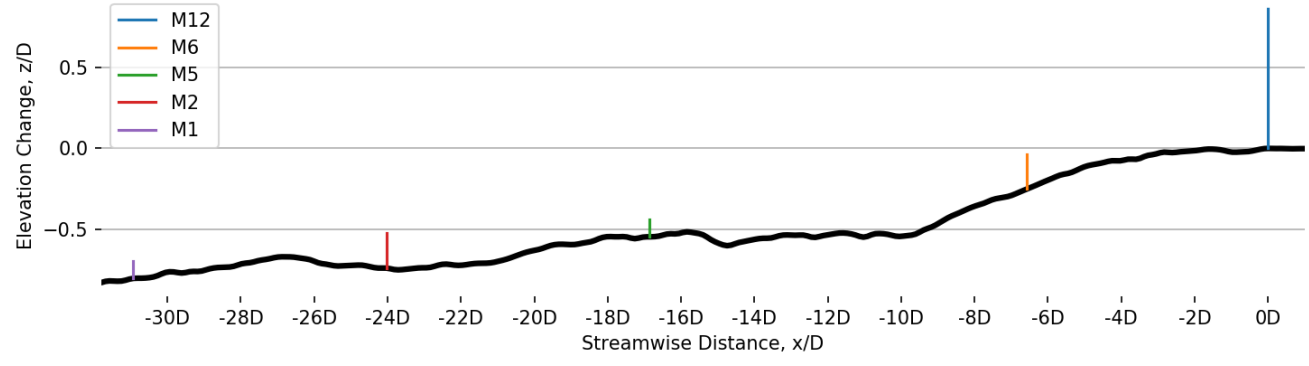

(c) WFIP2 Physics Site terrain relative to M12, with met masts represented by vertical lines

Figure 3. Terrain transects through simulated wind turbines and met masts; note the different scale in (c).

after the down-ramp at 4 p.m. local time. When used to diagnose stability, the shear exponent and TI tell different stories: the low shear suggests a well-mixed boundary layer (characteristic of unstable conditions), whereas the decrease in TI starting at noon local time suggests a transition toward atmospheric stability. However, the atmospheric stability state is not obvious when considering TI alone. TKE is a better indicator of stability than TI because it is an absolute measure [18, and is in better agreement with $z / L$. Therefore, from TKE and $z / L$ (Fig. 6), as well as the heat flux (Fig. 4), the conditions of the ABL during the study period describe the lead-up to a nocturnal transition, during which near-neutral conditions are characterized by atypically low TI.

\section{Terrain-Resolving Methodology}

The validation effort involves three models of increasing fidelity: FLOw Redirection and Induction in Steady State (FLORIS) [19], FAST.Farm [20, and the Simulator fOr Wind Farm Applications (SOWFA) [15]. SOWFA is a high-fidelity model of wind plant aerodynamics using LES, whereas FLORIS is an engineering wake model for controls applications. FAST.Farm is a midfidelity multiphysics approach that couples the turbulent flow in a wind farm with the structural response of the constituent wind turbines. Wake modeling 

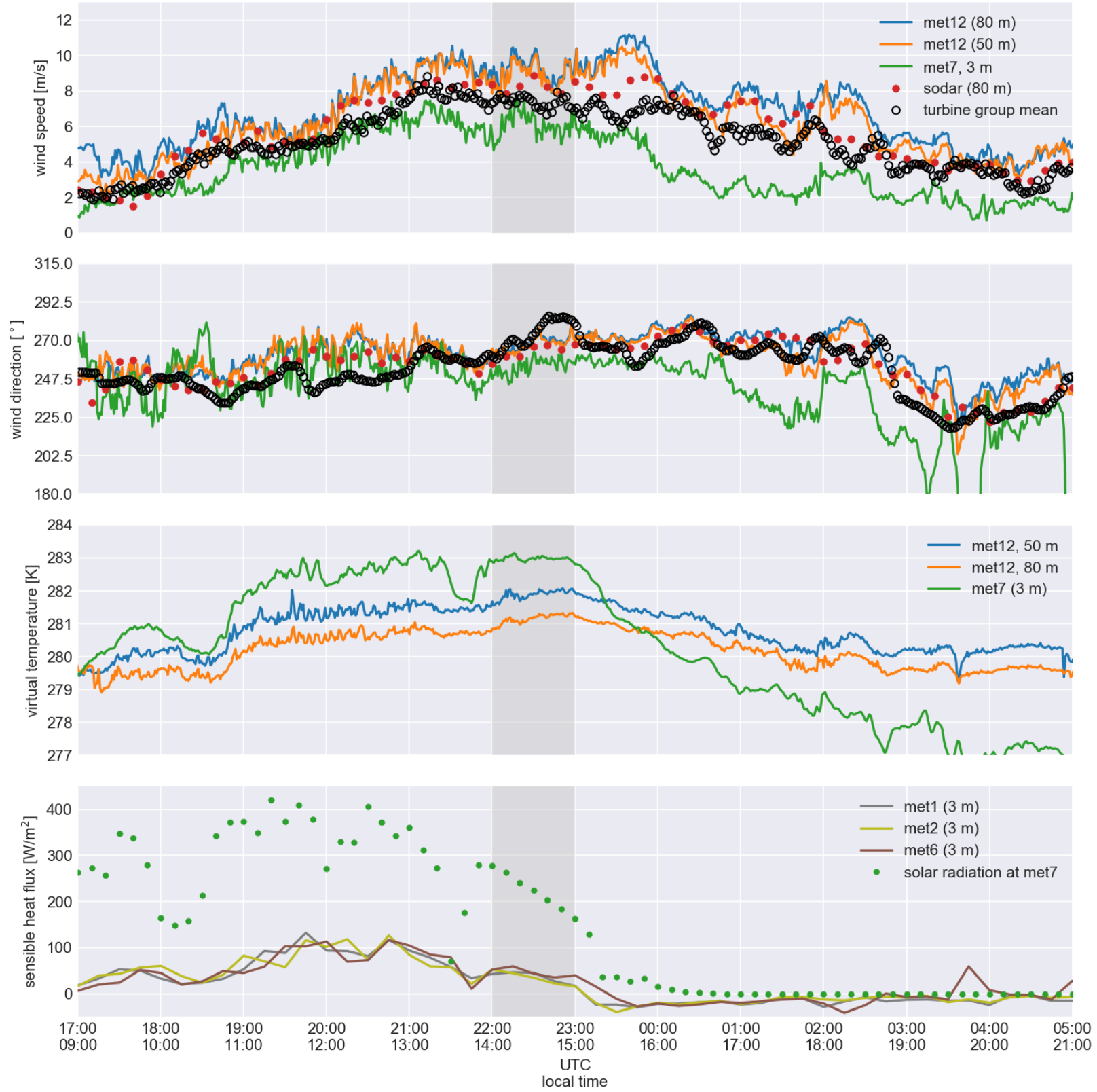

Figure 4. Observed wind speed, direction, temperature, and heat flux; the 1-hour period of interest is shaded.

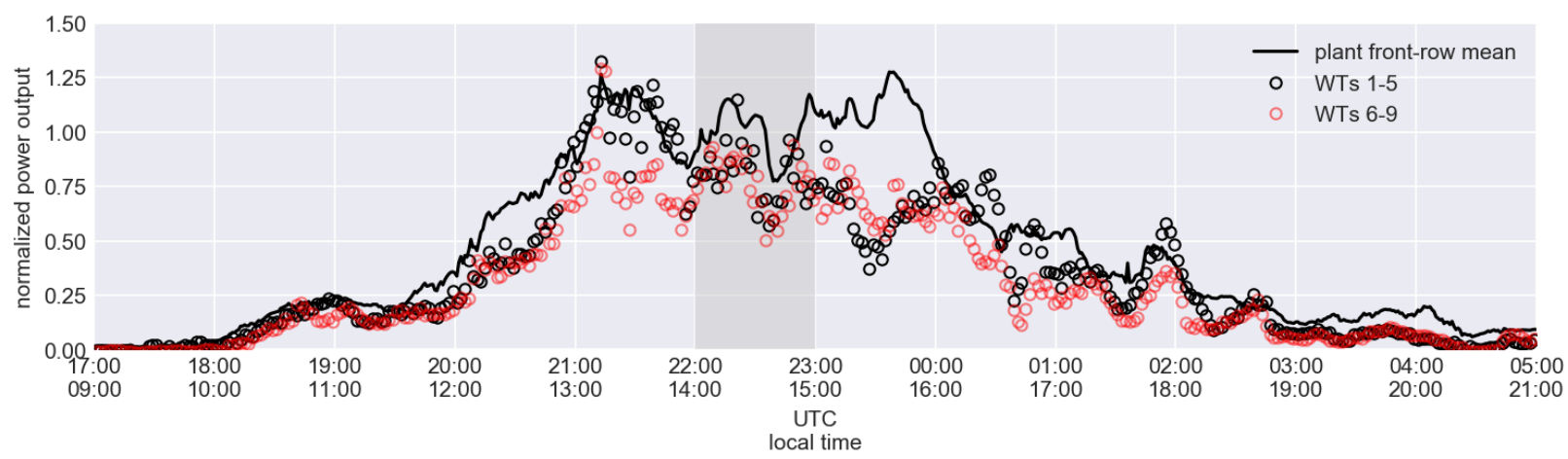

Figure 5. SCADA power data for selected wind turbines (WTs), normalized by the mean plant power during the (shaded) period of interest. 

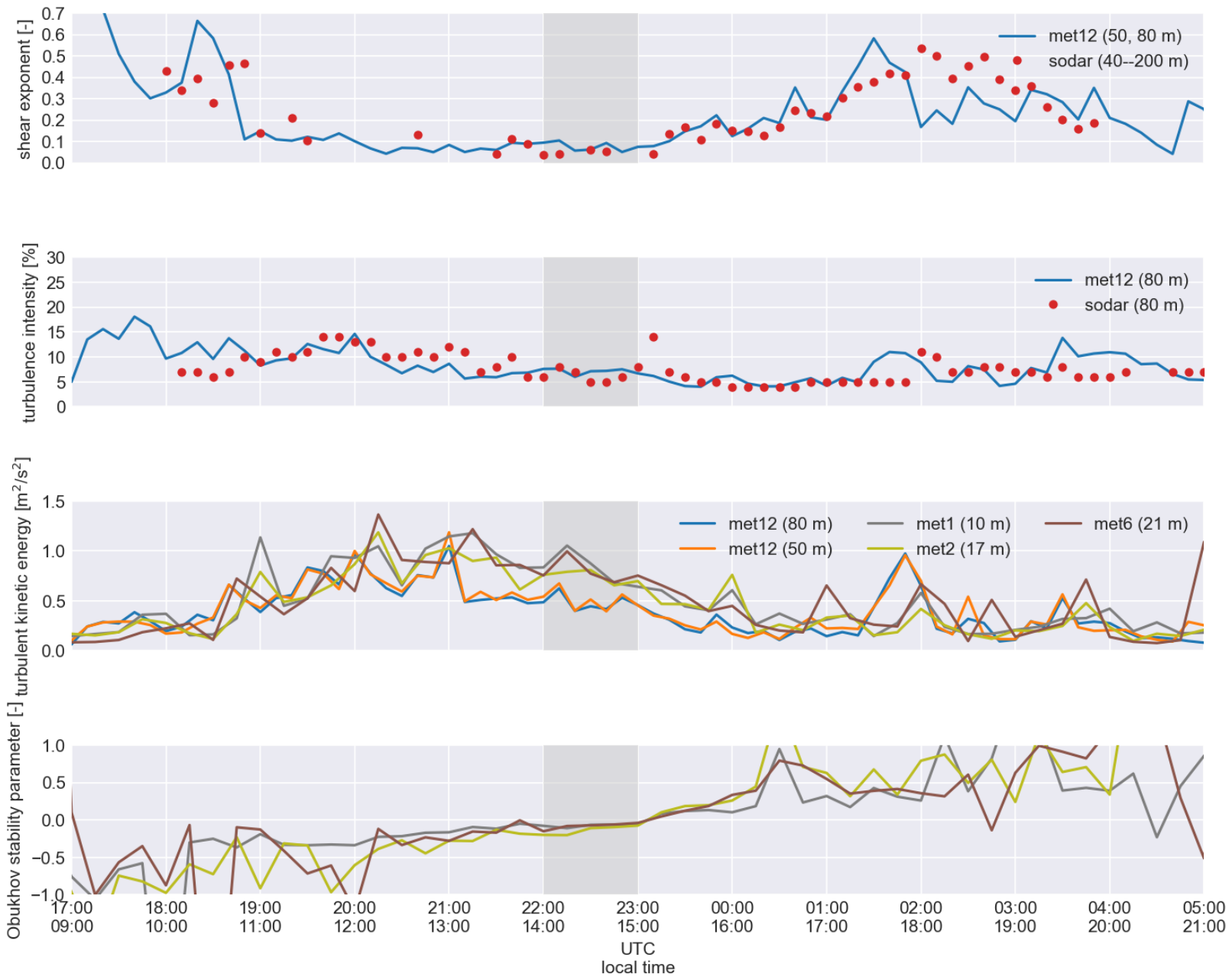

Figure 6. Ten-min averages of calculated atmospheric quantities.

parameters in FLORIS are tuned using flat-terrain SOWFA LES. To handle the complex terrain in this effort, we have modified FLORIS and FAST.Farm to account for local terrain-induced vertical velocities.

\section{III.A. FLORIS}

For this study, FLORIS [19 has been modified based on the model presented in Ref. 21]. In particular, the wake trajectory and recovery are modified when a wake goes up a hill based on the change in pressure gradient induced by the hill. Flow on the lee side of the hill is unchanged in the current modification. However, FLORIS does not take into account the spanwise and vertical velocities when computing wake deficits. Instead, FLORIS only uses the streamwise component and computes the wake deficits for one wind direction, i.e., there are no local variations in wind direction across the wind farm. Future versions of FLORIS will incorporate the spanwise and vertical velocities (as shown in Ref. 22]). In this study, both a uniform log-law wind profile (the default inflow) and a prescribed time-averaged velocity field from terrain-resolved LES have been considered.

\section{III.B. FAST.Farm}

FAST.Farm seeks to balance accurate flow and wind turbine multiphysics modeling with economical computational costs 20. That balance is designed specifically to support iterative design processes and system-wide optimization. FAST.Farm relies on OpenFAST 23 to calculate the aeroelastic response of each wind turbine in a wind power plant to an incoming flow field, which is supplied in this case by the terrain-resolving 
SOWFA precursor simulation. For the current study, the FAST-modeled structural degrees of freedom have been disabled to focus on aerodynamic interactions. The aerodynamic response, based on blade-element momentum theory, is then used to estimate the dynamic flow field in the wake of each turbine, which is advected downstream based on dynamic wake meandering principles. The wake flow from FAST.Farm is moved downstream according to an effective advection velocity, calculated within a series of planes parallel to the rotor. Turbine loads are calculated in near-rotor subdomains while wake meandering and merging calculations are calculated on a background domain. Any point excluded from the background domain (e.g., those points that are below the terrain surface) do not contribute to the effective advection velocity. By interpolating the LES field onto only points above the surface of the ground, the advection velocity is naturally terrain-following. The resulting turbulent wake flow does not pass through the surface, even though FAST.Farm does not include any explicit models for complex terrain, flow recirculation or separation, or local pressure gradients.

FAST.Farm simulations depend on two sampled data sets provided by a SOWFA large-eddy simulation. First, low-frequency sampling was performed every $2 \mathrm{~s}$ over a region encompassing all turbines of interest and extending downstream far enough so that simulated wake planes - the number of which is specified a priorido not advect out of the sampled domain. A second, high-frequency data set was sampled concurrently at $1 / 3$-s intervals in $2 D \times 2 D \times 2 D$ cubes centered around each turbine. This data set is used to calculate turbine power and unsteady aerodynamic loads.

\section{III.C. SOWFA Large-Eddy Simulation}

High-fidelity wake simulation is accomplished with SOWFA large-eddy simulation, which can accurately resolve all the energetic scales of turbulent motion in the ABL in addition to capturing the physics of wake meandering, expansion, and recovery. The standard SOWFA simulation approach first simulates a canonical ABL on a horizontally periodic domain. Turbulent flow from this precursor LES is then mapped to a timevarying Dirichlet boundary condition on a finite-domain simulation with turbines. Turbines are modeled as actuator lines that correspond to momentum sources [15. In this complex terrain study, we take the same approach to turbulent inflow generation as with flat terrain. Inflow planes are extracted from the flatterrain precursor and mapped onto the terrain-following mesh at the inlet boundary, in the same manner as Refs. 3, 24. This is a straightforward approach to generating inflow turbulence over complex terrain without having to enforce periodicity. Since the inflow does not have any resolved terrain-added turbulence, the flow will require some finite distance to adjust to the terrain. Over flat terrain, transition from under-resolved turbulence on a $20-\mathrm{m}$ grid to fully resolved turbulence on a $10-\mathrm{m}$ grid can require $1-2 \mathrm{~km} 25$. In complex terrain, we expect this transition to be facilitated by topographical features.

The precursor simulation is aimed at reproducing the conditions observed in the vicinity of the turbines at the WFIP2 Physics Site. Specifically, the high-frequency data from M12 were used to derive the mean flow conditions (from Fig. 4) and turbulence characteristics (from Fig. 6) at hub height, which were assumed to be stationary over the 1-hour study period between 2200 and 2300 UTC. Surface roughness, an input to the Schumann-Grötzbach surface shear-stress model, was used as a tuning parameter to obtain the desired shear exponent $(<0.1)$ and turbulence intensity at hub height $(5 \%-10 \%)$. At the lower surface, the measured heat flux (Fig. 4) was specified as a fixed flux boundary condition to provide heating representative of the slightly unstable, near-neutral ABL that existed in the period prior to the nocturnal transition. Turbulence closure is achieved with the Deardorff one-equation eddy-viscosity model. The only unknowns are the temperature profile and consequently the ABL height. Due to limited data availability at the Physics Site, the ABL height was estimated from the signal-to-noise ratio in measurements recorded by a radar acoustic sounding system located 3 miles away in Wasco, Oregon. During the study period, the temperature was assumed constant with height, a characteristic of near-neutral conditions, through the ABL up to an estimated ABL height of $600 \mathrm{~m}$.

The precursor computational domain has uniform grid spacing of $20 \mathrm{~m}$ up to $1 \mathrm{~km}$ in height, after which the vertical spacing increases with height at a growth rate of $5 \%$. The horizontal spacing is kept constant until the vertical spacing doubles, at which point the horizontal spacing also increases by a factor of two to match the vertical spacing. Overall, the mesh is coarsened in this manner up to a uniform cell size of $80 \mathrm{~m}$ and this cell size is extended up to a height of $3 \mathrm{~km}$, resulting in 4.1 million computational cells. A solver-adjustable time-step size was permitted with the condition that the maximum Courant number remains below 0.75 .

In the terrain-resolving, finite-domain simulation, the mesh is refined by cell splitting to $10 \mathrm{~m}$ within the 
outlined blue region in Fig. 2 to provide higher-resolution sampled fields for FLORIS and FAST.Farm. This approach results in a mesh with 7.7 million computational cells. A Laplacian mesh-motion solver [26] is used to stretch the mesh over terrain mapped by the Shuttle Radar Topography Mission, with the minimum domain height being $3 \mathrm{~km}$, corresponding to the precursor domain. The redistribution of points is controlled by an inverse-distance diffusivity term. To maintain numerical stability in the flow simulation on the resultant mesh, 1/6-s time steps were simulated with a maximum Courant number of 0.3 . Inflow to the lower-fidelity models was sampled from this simulation, ignoring the first 10 minutes of simulated time to account for terrain-induced transients.

After running the flat-terrain precursor, the simulation domain was further refined in the outlined green regions of Fig. 2. after three refinement steps, the finest grid spacing is $1.25 \mathrm{~m}$ around the rotor. This cell size allows for over 70 cells across the rotor diameter to provide sufficient resolution for actuator line modeling. The turbine models in SOWFA and FAST.Farm are both based on manufacturer-provided aerodynamics, structural, and controls data. Overall, the final terrain- and turbine-resolving mesh contains 92 million computational cells. The actuator-line simulation required a time-step size of $0.04 \mathrm{~s}$ that resulted in a maximum Courant number of 0.5.

\section{Results}

Results from the three models are presented in the following four sections. First, we assess how well the LES inflow matches the observed flow (Section IV.A). Next, we validate the power predicted by the three models against SCADA output (Section IV.B. Then, we analyze the flow field to explain our power predictions (Section IV.C). Finally, we characterize the different rotor wake trajectories over terrain (Section IV.D. Steady-state results from the FLORIS engineering model accounting for terrain were calculated in minutes on a personal computer. Midfidelity FAST.Farm results were obtained with a computational cost of $2 \mathrm{~s}$ wall clock time per second of simulation time on a 24-core high-performance-computing server. In comparison, the high-fidelity LES required 3 minutes of wall clock time per second of simulation time while utilizing 2064 cores (i.e., 100 core-hours per second of simulation time).

\section{IV.A. Simulated Flow Characteristics}

The validation against SCADA data first requires an accurate simulation of the background turbulent flow. Flow over flat terrain was simulated for 20,000 s to ensure that planar-averaged statistics are converged over time. Figure 7 shows the simulated quantities of interest after convergence: friction velocity, wind shear exponent, TI, and TKE. Simulated quantities from the flat, horizontally periodic precursor (black curves) are compared with virtual instruments sampled within SOWFA (color curves). With the flat precursor, statistical quantities were calculated in two ways, either from planar averages (assuming homogeneity) or from time averages of a point probe (assuming stationarity). The latter approach replicates the treatment of observations from the field. In all plots, the shaded region represents the range of values observed in the corresponding real-world instruments from WFIP2.

In lieu of directly specifying a velocity profile, which is not well defined given limited height-varying data, the mean hub-height velocity was kept fixed and surface roughness was adjusted to match the observed surface shear stress as described by friction velocity $u_{*}=\left[\left\langle u^{\prime} v^{\prime}\right\rangle^{2}+\left\langle u^{\prime} w^{\prime}\right\rangle^{2}\right]^{0.25}$. Prime quantities denote fluctuations about a mean and $\langle\cdot\rangle$ denotes time averaging. A surface roughness of $0.005 \mathrm{~m}$ in SOWFA results in a planar-averaged $u_{*}$ that matches the measurement mean. Unlike the planar average, however, the 10min time statistics of the flat-terrain probe displays significantly more variability. The mean $u_{*}$ is $9 \%$ lower than the planar average, but still within the range of observed values. Probes from the terrain-resolving simulation are lower than observations by $21 \%-34 \%$. However, because of terrain variations, the quantities of interest calculated from terrain-resolved probes are not expected to match the corresponding flat-terrain values.

More important than matching $u_{*}$ at the ground is to match the shear across the rotor and turbulence level at hub height, as these quantities have a much greater impact on turbine performance and loads. The estimated shear from the virtual sodar at M1 and virtual met mast at M12 are 0.022 and 0.032 , respectively. The M12 shear closely matches the precursor value because the precursor target values were specified based on the average values at this location. This difference may be attributed to the change in mean flow between M01 and M12, likely because M1 is located at 70-m lower elevation. 


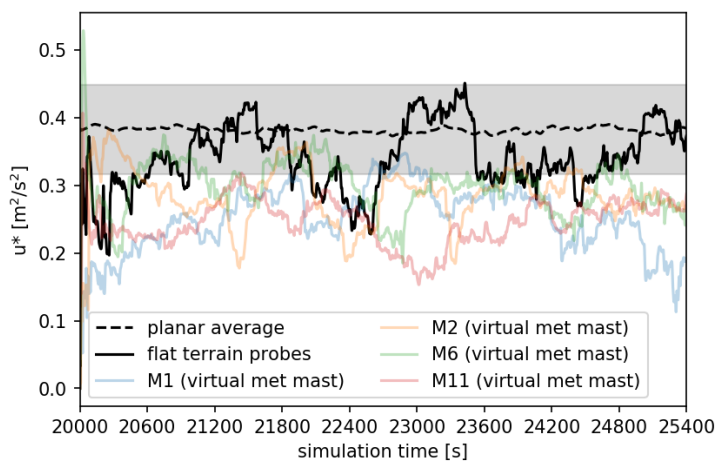

(a) Friction Velocity (at $z=10 \mathrm{~m}$ )

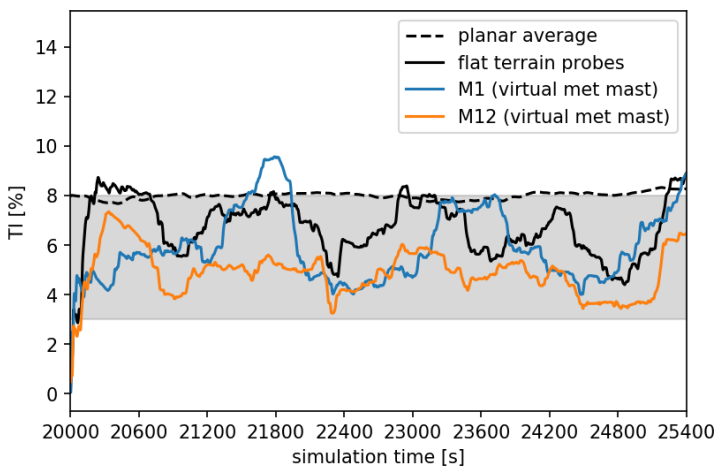

(c) Turbulence Intensity

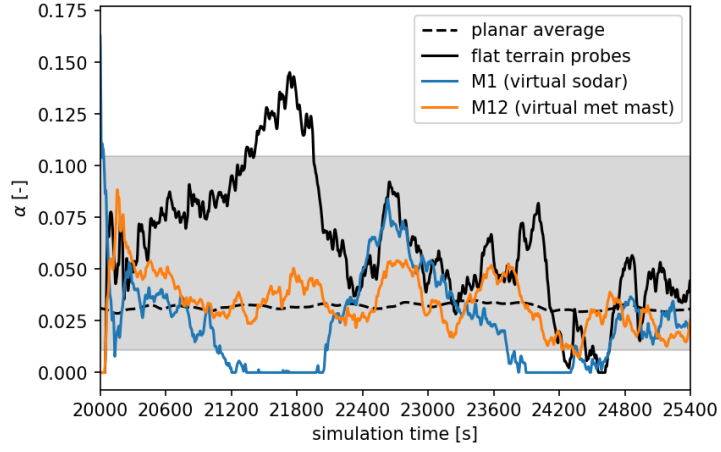

(b) Wind Shear

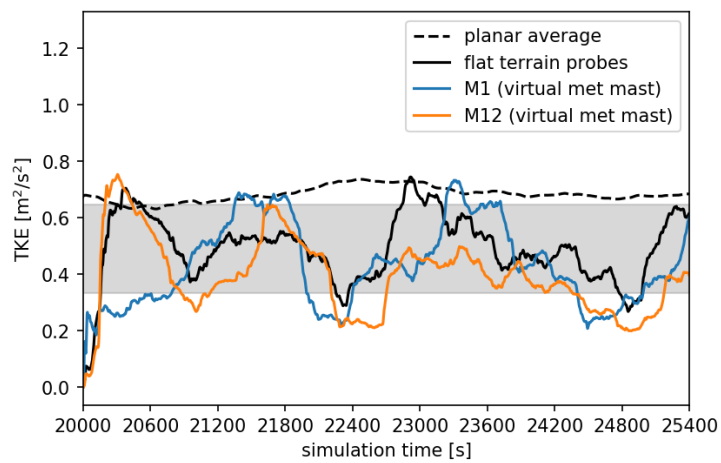

(d) Turbulent Kinetic Energy

Figure 7. Simulated conditions from the flat precursor simulation (black curves) and terrain-resolving simulation (color curves); the shaded region represents the range of observed values corresponding to the virtual measurements in terrain.

Turbulence levels in terms of TI and TKE are in agreement between all probe calculations, regardless of terrain. This agreement suggests that in this case, the modest terrain variation (in terms of elevation change and steepness) do not add an appreciable amount of turbulence and the primary terrain effect is on the mean flow. The mean and range of simulated values in both turbulence quantities is in excellent agreement with the observations at WFIP2. In addition, the velocity variances, which contribute to TI and TKE, are generally overpredicted by the planar-averaged statistics and should not be used for comparison with observations.

\section{IV.B. Average Power Production}

Having verified that our LES flow fields are representative of observed conditions at WFIP2 during the period of interest, we can now make an assessment of the power predictions for our three wind plant models. Figure 8 illustrates the mean and standard deviation (where applicable) in power for the reference SCADA data and model outputs. Power has been normalized by the mean power from the front-row turbines, WT15 (Fig. 2). Because FLORIS (with and without terrain) is a steady-state model, there is no measure of variability available. SCADA data are available at 2-min intervals, therefore, the power statistics are based on 30 data samples. In contrast, FAST.Farm and SOWFA have instantaneous power output at $100 \mathrm{~Hz}$ and $25 \mathrm{~Hz}$, respectively. Because we assumed that the period between 2200 and 2300 UTC is stationary, the reference SCADA data in both subplots are identical.

In total, 1.5 hours of terrain-resolved LES flow-field data (Fig.2) are available for FLORIS and FAST.Farm. Omitting start-up transients in the LES terrain simulation retains at least 1 hour of usable inflow data, which permits analysis of averaging effects. However, only 9 minutes of comparable SOWFA LES turbine simulation time are available because of computational resource limitations, so the high-fidelity results have been excluded from this analysis. Figure 8 illustrates the predicted power, neglecting the initial 10 minutes of each turbine simulation to allow for wake development. We then compare the effects of averaging over 10 
minutes - a standard averaging period in wind engineering practice - with a full 1-hour average to match the reference period. Note that the 10-min period in Fig. 8(a) overlaps with the first 10 minutes of the data contributing to the 1-hour results in Fig. 8(b). To replicate the SCADA data processing, the 1-hour FAST.Farm data were first resampled to 2-min intervals before calculating the mean and standard deviation. Resampling of the SCADA data was not performed for the 10-min analysis, as only 5 data points would have been retained and therefore precluded any statistically meaningful conclusion.

The full-hour average shows reduced variability in the FAST.Farm power output in terms of standard deviation. However, this variability metric ranges from $15 \%-32 \%$ of the mean in the SCADA data, likely resulting from data outliers and the limited sample size. As an alternative, the robust coefficient of variation $(\mathrm{RCoV})$ may be considered:

$$
\mathrm{RCoV}=\frac{\operatorname{median}(|P-\operatorname{median}(P)|)}{\operatorname{median}(P)}
$$

By this metric (indicated by the symbols in Fig. 8), the change in variability between turbines is negligible for both the SCADA and FAST.Farm data. The variability in the FAST.Farm predictions is within $11 \% \pm 2 \%$ of the median, whereas the variability in measured power is within $16 \% \pm 2 \%$ of the median.

Comparisons between the 10-min and 1-hour averaged power output indicate that a longer averaging period generally reduces the predicted change in power output resulting from terrain. In other words, the FLORIS results including terrain effects, which depend on the inflow averaging period, tend toward the flat-terrain FLORIS results (with log-law-modeled inflow) that have no time dependence. The front-row output tends toward unity for the 1-hour average for both models. For the 10-min period, the mean power for WTs $1-5$ is within $4 \%-15 \%$ of the front-row mean (i.e., a normalized turbine power of 1.0 ), and within $0 \%-6 \%$ for the 1 -hour period.

Simulated terrain effects on turbine power performance, based on Fig. 8(b), may be summarized into the following terrain cases:

1. Terrain modeling may reasonably predict turbine performance in the field (WTs 2, 4, and 8).

2. Terrain modeling overpredicts turbine performance, with FLORIS and FAST.Farm in agreement (WTs 1 and 3).

3. Terrain modeling underpredicts turbine performance, with FLORIS and FAST.Farm in agreement (WT5).

4. Terrain modeling underpredicts power production, with FLORIS and FAST.Farm in disagreement (WTs 6, 7, and 9).

Predictions are considered reasonable (Terrain Case 1) where the mean and/or median values are in agreement between the SCADA and model predictions. For Terrain Cases 1-3, FAST.Farm and FLORIS with terrain are in agreement in the front row (WTs 1-5). For all turbines in the back row (WTs 6-9), FAST.Farm and FLORIS differ significantly. Except for WT6, FLORIS with terrain results in negligible increases in power output in the back row.

The effectiveness of the models in Terrain Case 1 is not definitive, considering that the mean and median values tell different stories. Moreover, Terrain Case 1 includes a variety of terrain, as illustrated in Fig. 3: a turbine on an uphill slope (WT2), a turbine on the top of a hill (WT4), as well as a turbine on a slight downhill slope (WT8). Overpredictions of turbine performance (Terrain Case 2) typically result from overestimating the effect of the favorable pressure gradient. With WT2 as an exception, this trend holds true in the current results - WT1 and WT3 both have a relatively steep average windward slope of $13^{\circ}$ (Fig. 3(a)). Despite WT2 having a similar average windward slope of $11^{\circ}$, performance is not overpredicted because the turbine is situated on the upslope rather than at a local elevation maxima.

Underpredictions in turbine performance (Terrain Cases 3 and 4) may not be directly attributed to the slope of the local terrain. With the exception of WT7, FAST.Farm turbine power predictions are in closer agreement with SCADA data than those from FLORIS (with and without terrain). This may be due to the unsteady prescribed inflow to FAST.Farm, and/or FLORIS neglecting the local flow field when advecting the modeled turbine wakes. The terrain-induced flow physics are discussed further in Section IV.C. 


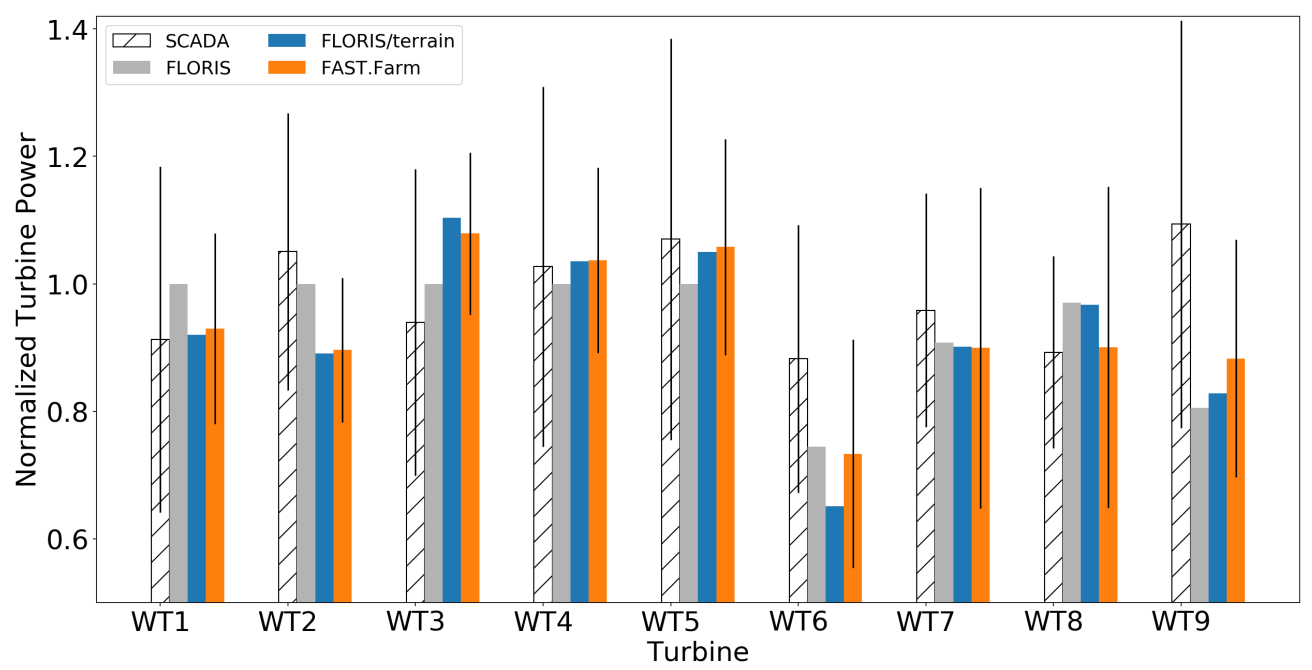

(a) 10-min average

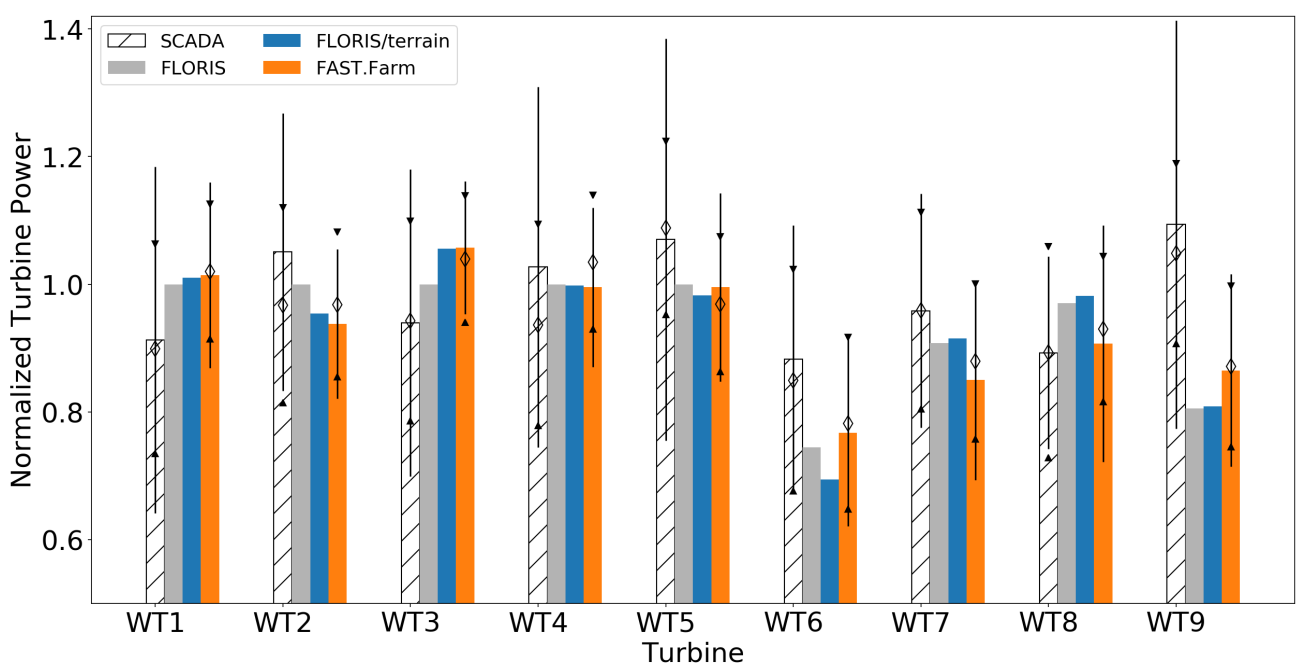

(b) 1-hour average

Figure 8. Comparison of measured and simulated mean normalized power output; vertical lines, open symbols, and filled symbols indicate the standard deviation, median, and robust coefficient of variation, respectively, for time-series data.

\section{IV.C. Flow-Field Analysis}

One of the key considerations for any wind power plant in a nonflat setting is the degree to which the terrain is expected to influence wake evolution and interaction. Terrain-conforming flow will exhibit local acceleration as the wind passes over (and around) the windward sides of hills and ridges, and local deceleration as the wind traverses over gullies and on the lee side of hills. Figure 9 illustrates the steady-state wakes predicted by FLORIS, based on a 1-hour average of the LES flow over the terrain. The performance of the front-row turbines matches the mid models (Fig. 8) because they have the same average inflow without any upwind turbine wakes. However, the current implementation of FLORIS neglects the local flow acceleration and deceleration caused by terrain, and assumes that the wakes are advected with the freestream. For this reason, FLORIS back-row predictions in Fig. 8, which include the front-row wakes, deviate from the FAST.Farm predictions that take into account for local terrain effects.

Figure 10 shows the average wind direction predicted by the LES without turbines, at $10 \mathrm{~m}$ above ground level, as a vector map superimposed over the velocity vector magnitude. The transverse $(y)$ component of the direction arrows has been scaled (by a factor of 10) to demonstrate the lateral flow deflection induced by the terrain. More prominent features, such as the gully on the inflow side of the domain, clearly induce 


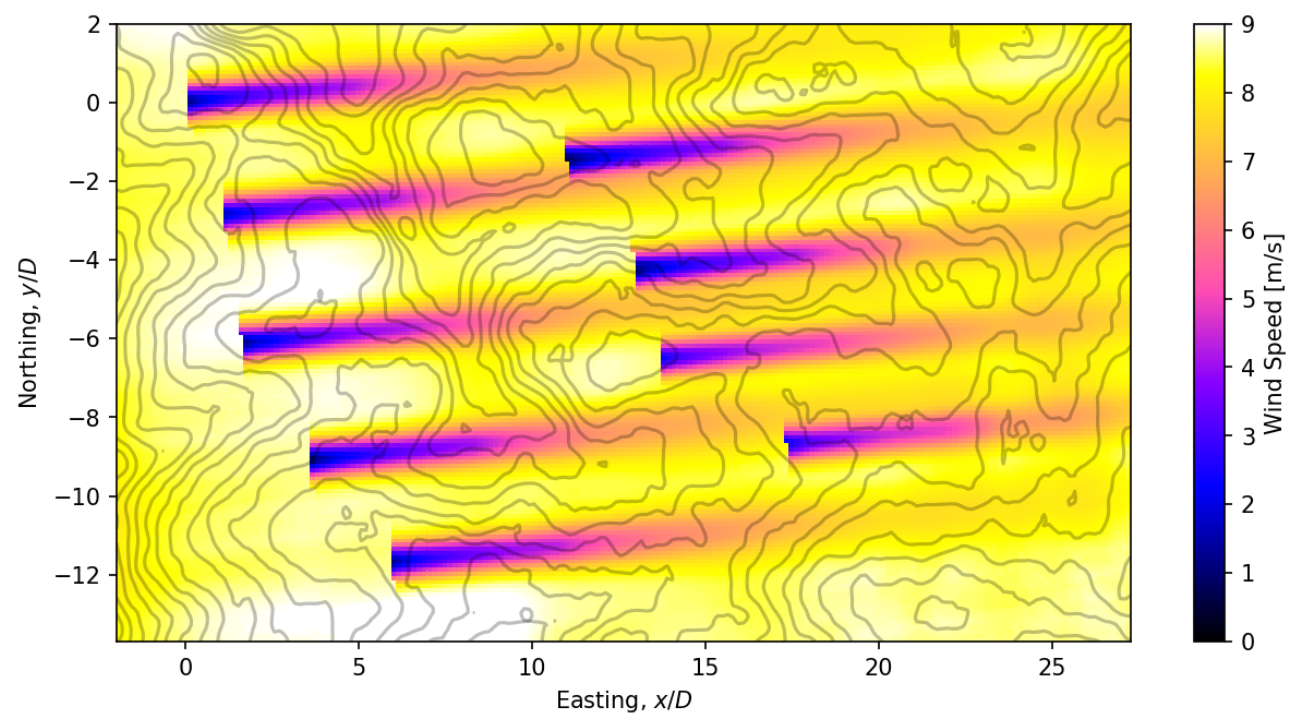

Figure 9. Steady-state FLORIS flow field at mean hub height, based on 1-hour-averaged inflow, with terrain indicated by contour lines (with elevation increasing toward the southeast).

strong changes in the local flow direction. Even in the absence of turbine wakes, WTs 6-9 operate in terraininduced velocity-deficit regions. In the case of WT 9, even though it operates in the wake of one or more upstream turbines, momentum recovery may be augmented by the terrain-following flow from the southwest (near coordinates $10 D,-15 D$ in Fig. 10 .

Lateral deflection of the background flow around terrain features will induce a similar lateral deflection in the trajectories of wind turbine wakes, at least in an instantaneous sense. For example, Fig. 11 shows an instantaneous velocity field illustrated by streamlines generated from within the gully mentioned above. Recalling that convergence or divergence of streamlines corresponds to flow acceleration and deceleration, respectively, it is clear that the terrain influences the atmospheric flow in a way that is favorable to WT 9, but disadvantageous to WT 8 . The green circles in Fig 11 are placed only to demonstrate the respective locations of each rotor disc; this LES velocity field does not include the actual wind turbines.

To estimate the degree to which the wake propagation direction is influenced by the terrain, deviation in aggregate flow direction is estimated as

$$
\phi_{\text {error }}(t)=\left\|\bar{\phi}-\phi_{\mathrm{WT}}(t)\right\|
$$

where $\phi$ is the wind direction, the overline implies time averaging, and $\|\cdot\|$ is the $L_{2}$-norm. The relative error of the wind direction ( $\phi_{\text {error }}$ ) within the FAST.Farm domain, a subset of the LES domain, is plotted in Fig. 12(a) This metric describes the deviation of the instantaneous wind direction (with turbines) from the time-averaged background wind direction (without turbines). The difference in mean wind-direction error between the flat-precursor and terrain-resolving background flow (dashed lines in Fig. 12(a)p indicates that the local terrain introduces a $5 \%$ bias to the flow direction on average. The minimum and maximum relative errors, indicated by the red points in Fig. 12(a), occur at times during which the flow with turbines deviates the least and most from the mean background flow, respectively.

Figure 13 shows the velocity vector magnitude on a surface at the mean hub height corresponding to these two cases. The SOWFA simulations with actuator-line turbines (Figs. 13(c) and 13(d) verify that the flow through the array over terrain is reasonably predicted by FAST.Farm. Note that the flow fields for the minimum deviation case (at $\mathrm{t}=21200 \mathrm{~s}$ ) are shown for both FAST.Farm and SOWFA, whereas for the maximum deviation case similar flow fields with large relative error were chosen. In the maximum deviation case, the meandering wakes expand the footprint of the array velocity-deficit region, as observed in both the mid- and high-fidelity models. The relationship between the maximum wind direction error and the array velocity deficit suggests that within these wake regions, the local wake dynamics dominate over the background topography.

Unsteady wake effects may be described by the wind-direction variation in a turbine wake. The precursor, terrain, and wind-power-plant flow fields were sampled at hub height, $2.5 \mathrm{D}$ upstream of WT 9 , in the wakes of 


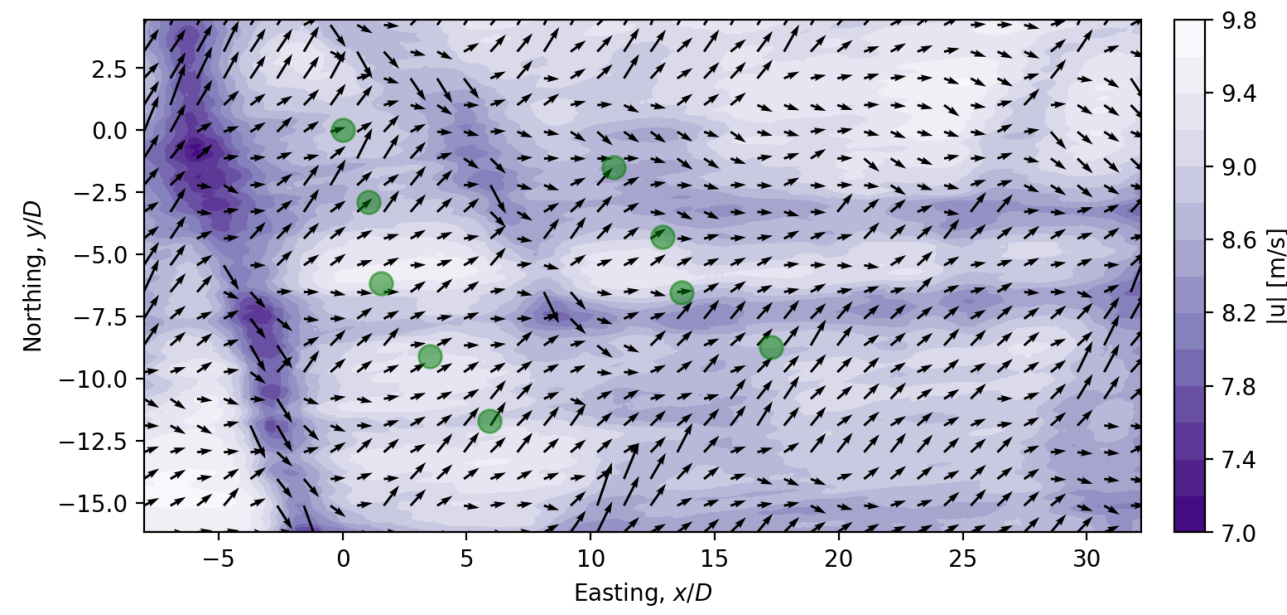

Figure 10. Velocity field calculated by terrain-resolving LES. Velocity direction vectors are superimposed on contours of velocity vector magnitude; the transverse vector component has been exaggerated to demonstrate terrain effects. The turbine locations (green circles) are not modeled but shown for reference.

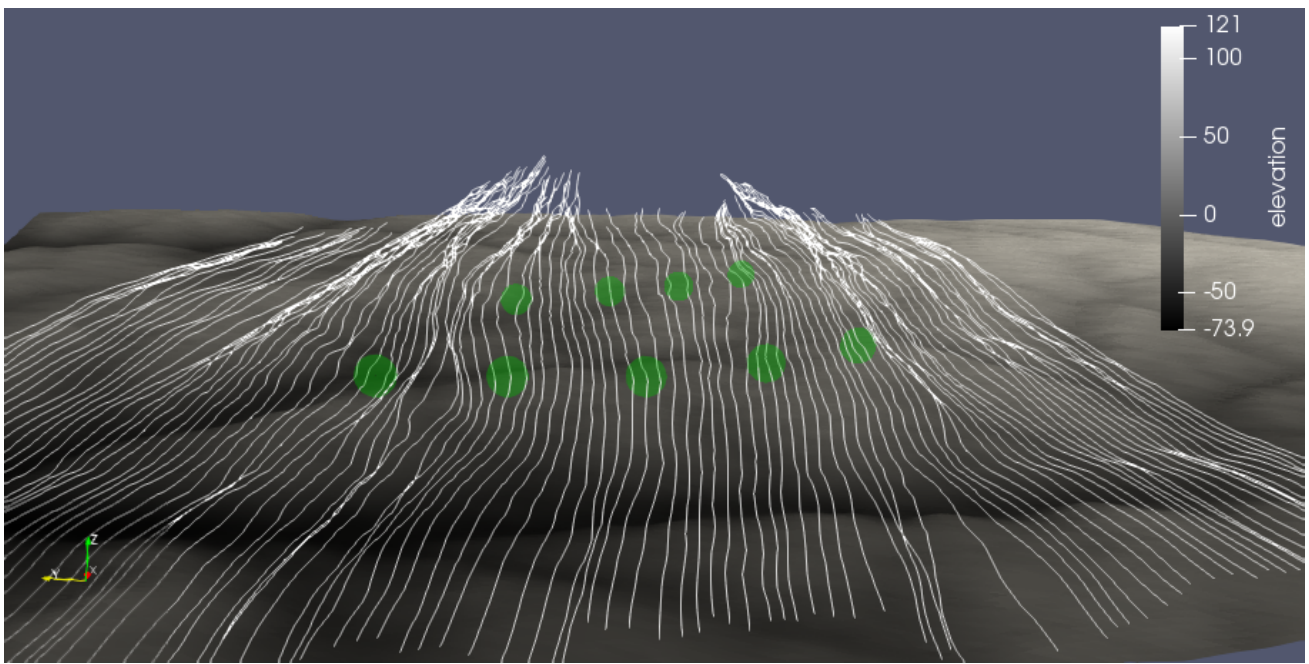

Figure 11. Representative streamlines depicting the instantaneous LES inflow to the group of turbines (shown for reference, not modeled, in this visualization). Change in elevation relative to WT 1 is shown. 
WTs 4 and 5. The time history of the standard deviation in wind direction at this probe is shown in Fig. 12(b). Compared to the flat-terrain case, the variability in the local flow over terrain is increased by a factor of 3 . The addition of turbines to the flow further increases the wind-direction variability intermittently, by $15 \%$ on average, from the terrain case without turbines. This increased wind-direction variability contributes to reduced back-row turbine performance.

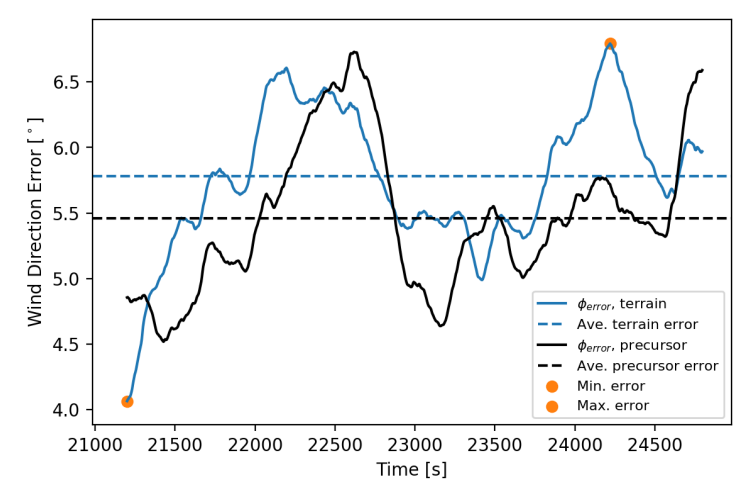

(a) Wind direction relative error $\phi_{\text {error }}$ between FAST.Farm flow field and background flow.

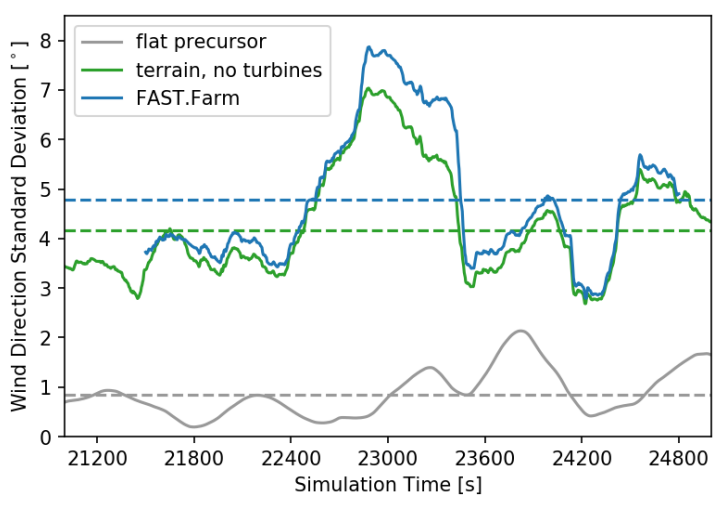

(b) Standard deviation of the simulated instantaneous wind direction from a hub-height probe.

Figure 12. Differences in simulated wind direction, with and without terrain.

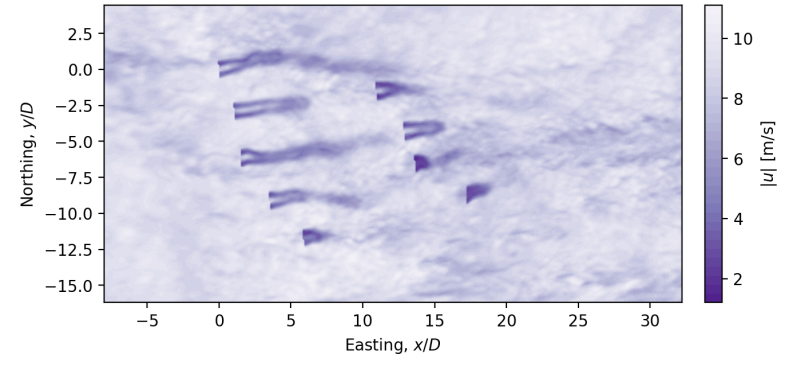

(a) FAST.Farm, $\mathrm{t}=21200 \mathrm{~s}$

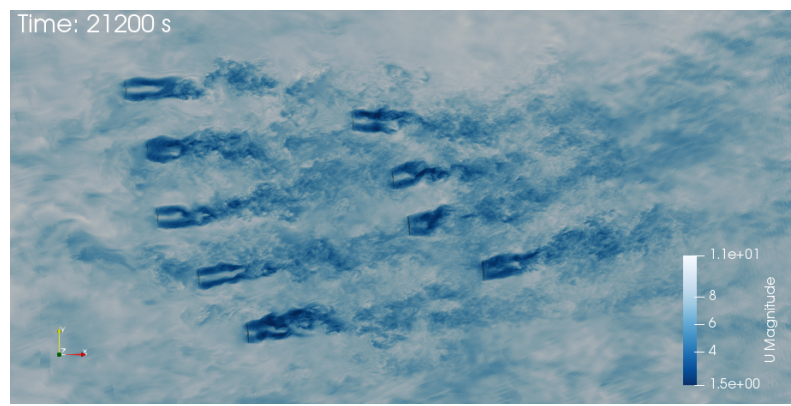

(c) SOWFA, $\mathrm{t}=21200 \mathrm{~s}$

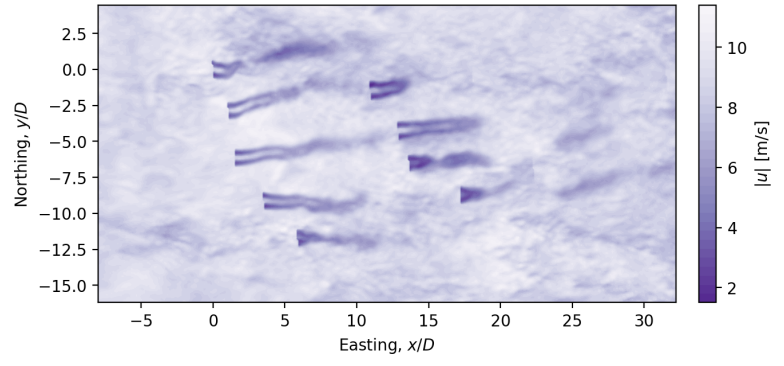

(b) FAST.Farm, t $=24200$

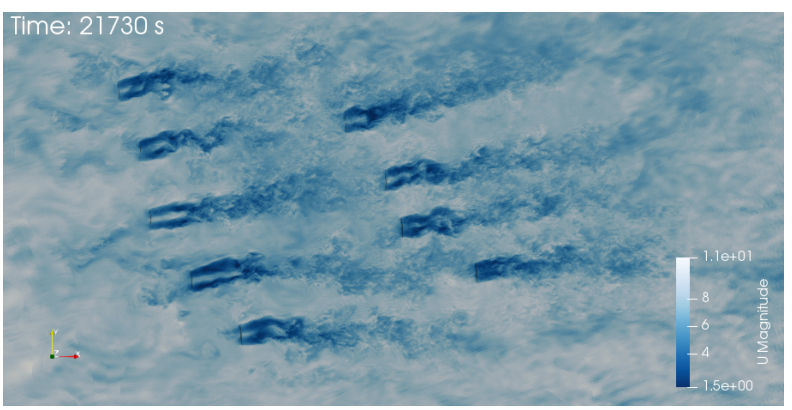

(d) SOWFA, t $=21730 \mathrm{~s}$

Figure 13. Instantaneous flow fields simulated by FAST.Farm and SOWFA (with actuator-line turbines), sampled at the average hub height of the nine turbines.

\section{IV.D. Wake Trajectories}

The FAST.Farm wake-advection model calculates the instantaneous wake center position for each simulated wind turbine at specified downstream distances. Having this information as output eliminates the need for a wake-identification strategy, which would add additional uncertainty to the wake analysis. Figure 14 reveals that, based on the FAST.Farm model output, the effects of the lee-side adverse pressure gradient on the vertical wake position are not negligible. 
Accurate prediction of turbine wake trajectories is crucial for accurate power predictions, especially with waked turbines. At $2 D$ downstream from each turbine, the wake position varies in the vertical direction by $0.1 D$. (In general, the wake is deflected upward due to rotor-shaft tilt away from the tower for upwind turbines.) By $4 D$, the range of wake heights has nearly tripled, ranging between approximately $0.2 D$ and $0.5 D$ above hub height. The influence of the terrain on the rotor wake is most clearly seen at $x<3 D$ downstream, where a downward slope clearly reduces the initial vertical wake displacement. For $x \geq 3 D$, the wake trajectory is less sensitive to large changes in terrain elevation. Further downstream, e.g., $x>6 D$, the influence of terrain appears negligible as the wakes appear to maintain their vertical trajectory.

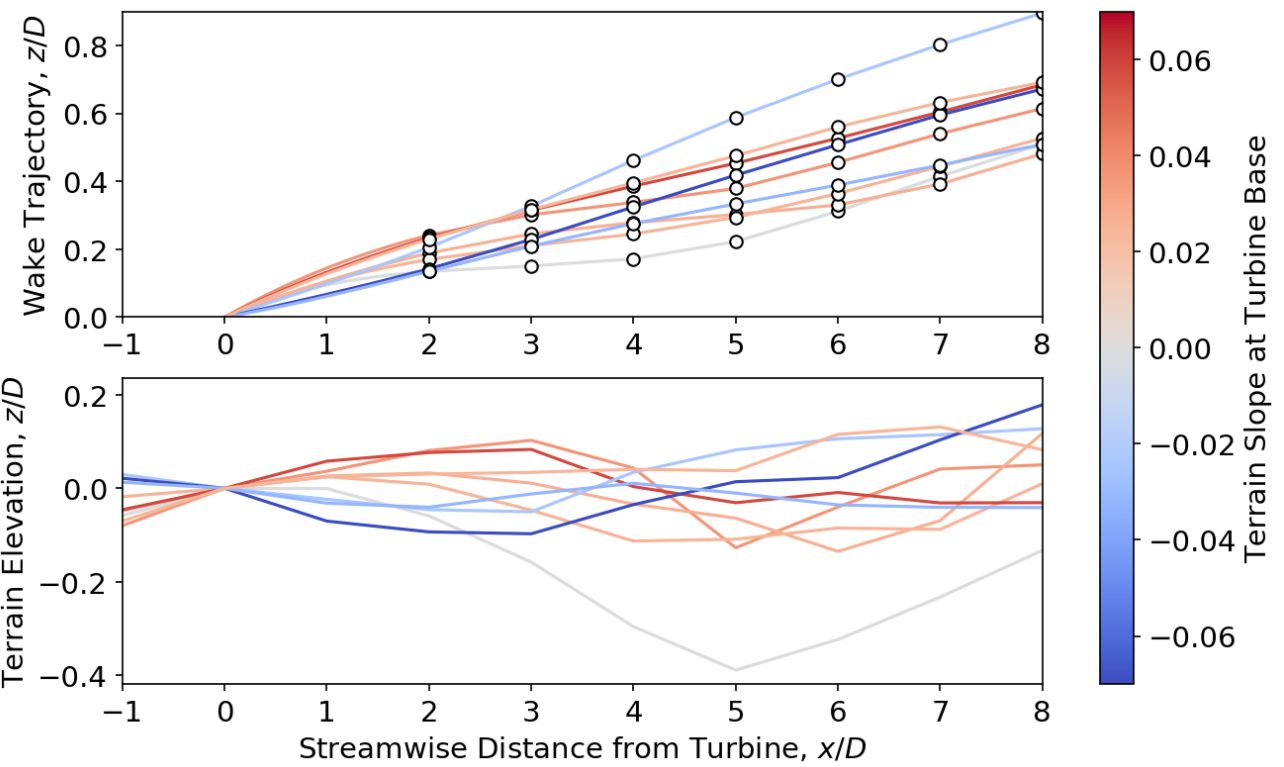

Figure 14. Time-averaged FAST.Farm wake trajectories (open symbols) and corresponding terrain slope, colored by the slope at the turbine base.

\section{Conclusions}

We have characterized the atmospheric conditions for a 12-hour period at the WFIP2 Physics Site, which is of interest to the wind energy community for a number of reasons: the large quantity and high quality of the inflow measurements, its proximity to multiple wind power plants, and the complex terrain and meteorology in the region. Within the selected day, a 1-hour period with atypical (low shear and low TI), near-neutral conditions has been identified. This period of interest occurs after a wind-speed ramp and immediately before a nocturnal ABL transition. A group of nine wind turbines have been analyzed during this period, highlighting the strengths of our models as well identifying areas for improvement.

One of the principal challenges in this study has been the appropriate treatment of spatial heterogeneity during the validation effort. Industry-standard 10-min averages may not include sufficient realizations of turbulence to recover a meaningful prediction of time-averaged turbine performance. By comparing the performance statistics of the nine turbines for a 10-min and 1-hour period, we found that errors in mean power for a 10-min period may be significant, varying up to $15 \%$ from the measured mean. Over the course of an hour, the variation in predicted mean front-row power was reduced by more than $50 \%$.

This case study revealed that power overprediction occurs for turbines placed on steeper slopes (modeled by both FLORIS and FAST.Farm), which agrees with previous findings in literature. The unsteady interactions between turbulent inflow, upstream turbine wake dynamics, and topography suggest that steady-state models may not accurately capture time-averaged turbine performance - even if reasonably long time averages are available. For FLORIS simulations including terrain, high-power-producing flow states may have been excluded as a result of averaging and/or neglected local flow. In comparison, FAST.Farm was able to capture more realistic interactions with the terrain and upstream turbine wakes, demonstrating improvements in power predictions for three out of four back-row turbines. In addition, the FAST.Farm results reveal that local terrain causes a bias in the wind direction field compared to freestream conditions, and 
significantly increases variability in the local wind direction. Both of these effects are expected to have an impact on turbine power production and loads. Even though only qualitative comparisons between SOWFA and FAST.Farm are possible at the moment, the preliminary SOWFA results suggest that FAST.Farm is able to capture realistic wake evolution over complex terrain. Future work will simulate the full hour of interest with actuator lines, enabling direct quantitative comparisons between all three models.

The value of FLORIS lies in its computational efficiency and applicability to wind-power-plant control and layout optimizations. Optimal control schemes and wind plant layouts determined by FLORIS will not necessarily be suboptimal, but conservative underpredictions of power are possible based on our findings regarding time averaging and unsteady effects. In addition, adverse pressure-gradient effects are not necessarily negligible, as we have found that the turbine wakes do have a tendency to follow downsloping terrain for up to $3 D$. Our FAST.Farm results suggest that an appropriate terrain-resolved inflow field can offer insights into the instantaneous power production of turbines within a wind plant, at significantly reduced computational cost compared to SOWFA, with mean power predictions that are as good as or better than FLORIS. However, the variability in power output is lower than the SCADA measurements. Without knowing the instantaneous yaw position of each turbine, the source of variability over the 1-hour period - either aerodynamics or yaw misalignment - will not be known with absolute certainty. In addition, FAST.Farm may overpredict the advection velocity as a consequence of the implemented averaging technique that accommodates terrain. By neglecting near-surface cells, the terrain elevation and near-surface flow velocity are artificially increased. This may contribute to the errors noted for turbines on relatively steep windward slopes.

\section{Acknowledgments}

Many thanks to Kelsey Shaler for her FAST.Farm support and to Emmanuel Branlard who developed the FAST model used in this effort. Also, to Patrick Hawbecker and Julie Lundquist, who provided guidance with interpreting the WFIP2 measurements.

This work was authored by the National Renewable Energy Laboratory, operated by Alliance for Sustainable Energy, LLC, for the U.S. Department of Energy (DOE) under Contract No. DE-AC36-08GO28308. Funding provided by the U.S. Department of Energy Office of Energy Efficiency and Renewable Energy Wind Energy Technologies Office. The views expressed in the article do not necessarily represent the views of the DOE or the U.S. Government. The U.S. Government retains and the publisher, by accepting the article for publication, acknowledges that the U.S. Government retains a nonexclusive, paid-up, irrevocable, worldwide license to publish or reproduce the published form of this work, or allow others to do so, for U.S. Government purposes.

\section{References}

${ }^{1}$ U.S. Department of Energy, "Wind Vision: A New Era for Wind Power in the United States," Tech. Rep. DOE/GO102015-4557, DOE Office of Energy Efficiency and Renewable Energy, Washington D.C., 2015.

${ }^{2}$ Fleming, P., Annoni, J., Scholbrock, A., Quon, E., Dana, S., Schreck, S., Raach, S., Haizmann, F., and Schlipf, D., "Full-Scale Field Test of Wake Steering," Wake Conference 2017, Visby, Sweden, May 2017.

${ }^{3}$ Yang, X., Pakula, M., and Sotiropoulos, F., "Large-Eddy Simulation of a Utility-Scale Wind Farm in Complex Terrain," Applied Energy, Vol. 229, Nov. 2018, pp. 767-777.

${ }^{4}$ Berg, J., Troldborg, N., Sørensen, N., Patton, E., and Sullivan, P., "Large-Eddy Simulation of Turbine Wake in Complex Terrain," Journal of Physics: Conference Series, Vol. 854, No. 012003, 2017.

${ }^{5}$ Quon, E., Churchfield, M. J., Cheung, L., and Kern, S., "Development of a Wind Plant Large-Eddy Simulation with Measurement-Driven Atmospheric Inflow," AIAA SciTech 2017, American Institute of Aeronautics and Astronautics, Grapevine, Texas, Jan. 2017.

${ }^{6}$ Wilczak, J., Finley, C., Freedman, J., Cline, J., Bianco, L., Olson, J., Djalalova, I., Sheridan, L., Ahlstrom, M., Manobianco, J., Zack, J., Carley, J. R., Benjamin, S., Coulter, R., Berg, L. K., Mirocha, J., Clawson, K., Natenberg, E., and Marquis, M., "The Wind Forecast Improvement Project (WFIP): A Public-Private Partnership Addressing Wind Energy Forecast Needs," Bulletin of the American Meteorological Society, Vol. 96, No. 10, Oct. 2015, pp. 1699-1718.

${ }^{7}$ Annoni, J., Fleming, P., Scholbrock, A., Roadman, J., Dana, S., Adcock, C., Porte-Agel, F., Raach, S., Haizmann, F., and Schlipf, D., "Analysis of Control-Oriented Wake Modeling Tools Using Lidar Field Results," Wind Energy Science Discussions, Feb. 2018, pp. 1-17.

${ }^{8}$ Wu, X., "Inflow Turbulence Generation Methods," Annual Review of Fluid Mechanics, Vol. 49, No. 1, 2017, pp. 23-49.

${ }^{9}$ Gopalan, H., Gundling, C., Brown, K., Roget, B., Sitaraman, J., Mirocha, J. D., and Miller, W. O., "A Coupled Mesoscale-Microscale Framework for Wind Resource Estimation and Farm Aerodynamics," Journal of Wind Engineering and Industrial Aerodynamics, Vol. 132, Sept. 2014, pp. 13-26. 
${ }^{10}$ Rodrigo, J. S., Arroyo, R. C., Gancarski, P., Guillén, F. B., Avila, M., Barcons, J., Folch, A., Cavar, D., D Allaerts, Meyers, J., and Dutrieux, A., "Comparing Meso-Micro Methodologies for Annual Wind Resource Assessment and Turbine Siting at Cabauw," J. Phys.: Conf. Ser., Vol. 1037, No. 7, 2018, pp. 072030.

${ }^{11}$ Politis, E. S., Prospathopoulos, J., Cabezon, D., Hansen, K. S., Chaviaropoulos, P. K., and Barthelmie, R. J., "Modeling Wake Effects in Large Wind Farms in Complex Terrain: The Problem, the Methods and the Issues: Wake Effects in Large Wind Farms in Complex Terrain," Wind Energy, Vol. 15, No. 1, Jan. 2012, pp. 161-182.

${ }^{12}$ Shamsoddin, S. and Porté-Agel, F., "Large-Eddy Simulation of Atmospheric Boundary-Layer Flow Through a Wind Farm Sited on Topography," Boundary-Layer Meteorology, Vol. 163, No. 1, April 2017, pp. 1-17.

${ }^{13} \mathrm{Ma}, \mathrm{Y}$. and Liu, H., "Large-Eddy Simulations of Atmospheric Flows Over Complex Terrain Using the Immersed-Boundary Method in the Weather Research and Forecasting Model," Boundary-Layer Meteorology, Vol. 165, No. 3, Dec. 2017 , pp. 421-445.

${ }^{14}$ NASA JPL, "NASA Shuttle Radar Topography Mission Global 1 Arc Second," 2013.

${ }^{15}$ Churchfield, M. J., Lee, S., Moriarty, P. J., Martinez, L. A., Leonardi, S., Vijayakumar, G., and Brasseur, J. G., "A Large-Eddy Simulation of Wind-Plant Aerodynamics," 50th AIAA Aerospace Sciences Meeting, Nashville, Tennessee, Jan. 2012.

${ }^{16}$ Haupt, S., Anderson, A., Berg, L., Brown, B., Churchfield, M., Draxl, C., Kalb, C., Koo, E., Kosovic, B., Kotamarthi, R., Mazzaro, L., Mirocha, J., Quon, E., Rai, R., and Sever, G., "Third-Year Report of the Atmosphere to Electrons Mesoscaleto-Microscale Coupling Project," Tech. rep., Pacific Northwest National Laboratory, Dec. 2017.

${ }^{17}$ Wharton, S. and Lundquist, J. K., "Atmospheric Stability Affects Wind Turbine Power Collection," Environ. Res. Lett., Vol. 7, No. 1, 2012, pp. 014005.

${ }^{18}$ Wharton, S. and Lundquist, J. K., "Assessing Atmospheric Stability and Its Impacts on Rotor-Disk Wind Characteristics at an Onshore Wind Farm: Atmospheric Stability on Rotor-Disk Wind Characteristics," Wind Energy, Vol. 15, No. 4, May 2012, pp. 525-546.

${ }^{19}$ Gebraad, P. M. O., Teeuwisse, F. W., van Wingerden, J. W., Fleming, P. A., Ruben, S. D., Marden, J. R., and Pao, L. Y., "Wind Plant Power Optimization through Yaw Control Using a Parametric Model for Wake Effects-a CFD Simulation Study," Wind Energ., Vol. 19, No. 1, Jan. 2016, pp. 95-114.

${ }^{20}$ Jonkman, J. M., Annoni, J., Hayman, G., Jonkman, B., and Purkayastha, A., "Development of FAST.Farm: A New Multi-Physics Engineering Tool for Wind-Farm Design and Analysis," 35th Wind Energy Symposium, American Institute of Aeronautics and Astronautics, Grapevine, Texas, Jan. 17.

${ }^{21}$ Shamsoddin, S. and Porté-Agel, F., "Wind Turbine Wakes over Hills," Journal of Fluid Mechanics, Vol. 855, Nov. 2018, pp. 671-702.

${ }^{22}$ Martínez-Tossas, L. A., Annoni, J., Fleming, P. A., and Churchfield, M. J., "The Aerodynamics of the Curled Wake: A Simplified Model in View of Flow Control," Wind Energy Science Discussions, Aug. 2018, pp. 1-17.

23 "OpenFAST Documentation," \url\{http://openfast.readthedocs.io/en/master/\}, Nov. 2017.

${ }^{24}$ Han, Y., Stoellinger, M., and Naughton, J., "Large Eddy Simulation for Atmospheric Boundary Layer Flow over Flat and Complex Terrains," Journal of Physics: Conference Series, Vol. 753, Sept. 2016, pp. 032044

${ }^{25}$ Quon, E. W., Ghate, A. S., and Lele, S. K., "Enrichment Methods for Inflow Turbulence Generation in the Atmospheric Boundary Layer," J. Phys.: Conf. Ser., Vol. 1037, No. 7, 2018, pp. 072054.

${ }^{26}$ Löhner, R. and Yang, C., "Improved ALE Mesh Velocities for Moving Bodies," Communications in Numerical Methods in Engineering, Vol. 12, No. 10, 1996, pp. 599-608. 